\title{
Approximations and inequalities for the exponential beta function
}

\author{
Silvestru Sever Dragomir ${ }^{1,2^{*}}$ and Farzad Khosrowshahi
}

\author{
"Correspondence: \\ sever.dragomir@vu.edu.au \\ ${ }^{1}$ College of Engineering \& Science, \\ Victoria University, Melbourne City, \\ Australia \\ ${ }^{2}$ DST-NRF Centre of Excellence in \\ the Mathematical and Statistical \\ Sciences, School of Computer \\ Science \& Applied Mathematics, \\ University of the Witwatersrand, \\ Johannesburg, South Africa
}

\begin{abstract}
In this paper, motivated by interest in simulating the expenditure patterns of construction projects, we introduce the mathematical concept of Exponential-Beta function by$$
F(\alpha, \beta):=\int_{0}^{1} \exp \left[x^{\alpha}(1-x)^{\beta}\right] d x
$$

where $\alpha, \beta$ are positive numbers. Taylor's-type approximations, several analytic inequalities, and global convexity properties are established.
\end{abstract}

MSC: $26 \mathrm{D} 15$

Keywords: Beta function; Gamma function; Taylor's formula; Analytic inequalities; Log-convexity; Global convexity

\section{Introduction}

The ability to accurately predict the client's financial commitment, which forms the basis of contractors' revenue, provides a pre-warning for alternative courses of action, which could prove detrimental to the success or, indeed, survival of both stakeholders. Cash flow mishaps account for considerable number of construction contractors' failures. The capital commitment locked in the expenditure flow of construction projects exerts considerable demand on the contractor to secure finance, particularly when the contractor is undertaking a number of projects simultaneously. To this end, an accurate forecast of project cash flow could be critical to the success and, indeed, failure of the firm. Ultimately, forecasting is a crystal gazing exercise, and the forecast of the expenditure is more challenging than the income side. Since the 1970s there have been several models developed by researchers and the industry. These models have been classified under different categories. One classification by Khosrowshahi and Kaka [8] consists of activitybased, element-based, and mathematics-based. While the elemental approach focuses on the time-related accumulation of cost centres (or elements), the activity-based approach consists of a laborious task of identifying and quantifying activity sequences, costs, and times. On the other hand, the mathematical approach offers a fast, cheap, and easy solution. While comparatively alienating to the user, it has the advantage of generating a forecast in early stages, and unlike other models, this approach requires much less information about the project.

(c) The Author(s) 2019. This article is distributed under the terms of the Creative Commons Attribution 4.0 International License (http://creativecommons.org/licenses/by/4.0/), which permits unrestricted use, distribution, and reproduction in any medium, provided you give appropriate credit to the original author(s) and the source, provide a link to the Creative Commons license, and indicate if changes were made. 
Extensive analysis of construction project expenditure patterns has revealed that the shape of the project periodic expenditure pattern is defined in terms of a number of variables represented by the following expression (see [7]):

$$
Y_{C}:=\exp \left[b x^{a}(1-x)^{d}\right]-1
$$

where

$$
x_{p}:=R=\frac{a}{a+d} \quad \text { and } \quad y_{p}:=Q=\exp \left[b R^{a}(1-R)^{d}\right]-1,
$$

with $Q$ and $R$ representing the positions of the project expenditure peak on both the cost and time access; $a$ and $b$ being parameterised in terms of $x_{p}$ and $y_{p}$ as follows:

$$
a=\frac{x_{p} d}{1-x_{p}}, \quad b=\frac{\ln \left(1+y_{p}\right)}{x_{p}^{a}\left(1-x_{p}\right)^{d}} .
$$

Parameter $d$ is calculated numerically and derived to rapidly converge towards a solution within desired error tolerance.

A relationship is established between the properties of the project and the physical shape of the project expenditure pattern. These are then related and reflected on the mathematical expression through its parameters.

Motivated by the above considerations, one can explore the mathematical behavior of the family of functions

$$
f_{\alpha, \beta}(x):=\exp \left[x^{\alpha}(1-x)^{\beta}\right], \quad x \in[0,1], \alpha, \beta>0
$$

and the "exponential Beta function" defined by the integral

$$
F(\alpha, \beta):=\int_{0}^{1} \exp \left[x^{\alpha}(1-x)^{\beta}\right] d x, \quad \alpha, \beta>0 .
$$

In order to establish the fundamental properties of these functions, we need some wellknown facts related to the classical Euler's Beta function summarised below.

\section{Some facts on beta function}

In mathematics, the Beta function, also called the Euler integral of the first kind, is a special function defined by

$$
B(\alpha, \beta):=\int_{0}^{1} t^{\alpha-1}(1-t)^{\beta-1} d t, \quad \alpha>0, \beta>0 .
$$

The utility of the Beta function is often overshadowed by that of the Gamma function, partly perhaps because it can be evaluated in terms of the Gamma function. However, since it occurs so frequently in practice, a special designation for it is widely accepted.

It is obvious that the Beta function has the symmetry property

$$
B(\alpha, \beta)=B(\beta, \alpha), \quad \alpha>0, \beta>0,
$$


and the following connection between the Beta and Gamma functions holds:

$$
B(\alpha, \beta)=\frac{\Gamma(\alpha) \Gamma(\beta)}{\Gamma(\alpha+\beta)}, \quad \alpha>0, \beta>0,
$$

where

$$
\Gamma(\alpha)=\int_{0}^{\infty} e^{-t} t^{\alpha-1} d t, \quad \alpha>0 .
$$

The following properties of the Beta function also hold (see, for example, [2, pp. 68-70]):

$$
\begin{aligned}
& B(\alpha+1, \beta)+B(\alpha, \beta+1)=B(\alpha, \beta), \quad \alpha, \beta>0 ; \\
& B(\alpha, \beta+1)=\frac{\beta}{\alpha} B(\alpha+1, \beta)=\frac{\beta}{\alpha+\beta} B(\alpha, \beta), \quad \alpha, \beta>0 ; \\
& B(\alpha, \alpha)=2^{1-2 \alpha} B\left(\alpha, \frac{1}{2}\right), \quad \alpha>0 ; \\
& B(\alpha, \beta) B(\alpha+\beta, \gamma) B(\alpha+\beta+\gamma, \delta) \\
& \quad=\frac{\Gamma(\alpha) \Gamma(\beta) \Gamma(\gamma) \Gamma(\delta)}{\Gamma(\alpha+\beta+\gamma+\delta)}, \quad \alpha, \beta, \gamma, \delta>0 ; \\
& B\left(\frac{1+\alpha}{2}, \frac{1-\alpha}{2}\right)=\pi \sec \left(\frac{\alpha \pi}{2}\right), \quad 0<\alpha<1 ;
\end{aligned}
$$

and

$$
B(\alpha, \beta)=\frac{1}{2} \int_{0}^{1} \frac{t^{\alpha-1}+t^{\beta-1}}{(t+1)^{\alpha+\beta}} d t=p^{\alpha}(1+p)^{\alpha+\beta} \int_{0}^{1} \frac{t^{\alpha-1}(1-t)^{\beta-1}}{(t+p)^{\alpha+\beta}} d t
$$

for $\alpha, \beta, p>0$.

In [5], Dragomir et al. obtained the following basic inequalities for the Beta function:

Theorem 1 Let $m, n, p, q$ be positive numbers with the property that

$$
(p-m)(q-n) \leq(\geq) 0
$$

Then

$$
B(p, q) B(m, n) \geq(\leq) B(p, n) B(m, q) .
$$

In particular, the following is true:

Corollary 1 For any $p, m>0$, we have the inequalities

$$
B(m, p) \geq[B(p, p) B(m, m)]^{\frac{1}{2}} .
$$

The positive real numbers $a$ and $b$ may be called similarly (oppositely) unitary if

$$
(a-1)(b-1) \geq(\leq) 0
$$


Theorem 2 Let $a, b>0$ be similarly (oppositely) unitary. Then

$$
B(a, b) \geq(\leq) \frac{1}{a b}
$$

We also have

Theorem 3 Let $m, n, p$ and $q$ be positive numbers. Then

$$
\begin{aligned}
& |B(m+p+1, n+q+1)-B(m+1, n+1) B(p+1, q+1)| \\
& \quad \leq \frac{1}{4} \frac{p^{p} q^{q}}{(p+q)^{p+q}} \frac{m^{m} n^{n}}{(m+n)^{m+n}} .
\end{aligned}
$$

Another simpler inequality that one can derive via Grüss' inequality is the following [5]:

Theorem 4 Let $p, q>0$. Then we have the inequality

$$
\left|B(p+1, q+1)-\frac{1}{(p+1)(q+1)}\right| \leq \frac{1}{4}
$$

or, equivalently,

$$
\max \left\{0, \frac{3-p q-p-q}{4(p+1)(q+1)}\right\} \leq B(p+1, q+1) \leq \frac{5+p q+p+q}{4(p+1)(q+1)} .
$$

We also have the following global convexity property; see, for instance, [5].

Theorem 5 The mapping $B$ is logarithmically convex on $(0, \infty)^{2}$ as a function of two variables.

For other properties of the Beta function, see the recent papers $[1,9,11]$ and the references therein.

\section{Basic facts on the generating function}

We consider the two-parameter generating function $f_{\alpha, \beta}:[0,1] \rightarrow[0, \infty)$,

$$
f_{\alpha, \beta}(x):=\exp \left[x^{\alpha}(1-x)^{\beta}\right]
$$

where $\alpha, \beta$ are positive constants. This family can be extended for negative numbers by eliminating either ends of the closed interval $[0,1]$. However, we do not consider this case here.

Define a simpler two-parameter family that generates the Beta function, $g_{\alpha, \beta}:[0,1] \rightarrow$ $[0, \infty)$, given by

$$
g_{\alpha, \beta}(x)=x^{\alpha}(1-x)^{\beta}
$$

where $\alpha, \beta$ are positive constants.

We start with the simple fact incorporated in the following: 
Proposition 1 Let $\alpha, \beta>0$. The function $f_{\alpha, \beta}$ is increasing on $\left[0, \frac{\alpha}{\alpha+\beta}\right]$, decreasing on $\left[\frac{\alpha}{\alpha+\beta}, 1\right]$, and

$$
\max _{x \in[0,1]} f_{\alpha, \beta}(x)=f_{\alpha, \beta}\left(\frac{\alpha}{\alpha+\beta}\right)=\exp \left[\left(\frac{\alpha}{\alpha+\beta}\right)^{\alpha}\left(\frac{\beta}{\alpha+\beta}\right)^{\beta}\right]
$$

Proof We have

$$
f_{\alpha, \beta}(x)=\exp \left[g_{\alpha, \beta}(x)\right]
$$

and

$$
f_{\alpha, \beta}^{\prime}(x)=g_{\alpha, \beta}^{\prime}(x) \exp \left[g_{\alpha, \beta}(x)\right], \quad x \in[0,1]
$$

showing that the sign of $f_{\alpha, \beta}^{\prime}$ on $[0,1]$ is the same with that of $g_{\alpha, \beta}^{\prime}$.

Further, we have

$$
\begin{aligned}
g_{\alpha, \beta}^{\prime}(x) & =\alpha x^{\alpha-1}(1-x)^{\beta}-\beta x^{\alpha}(1-x)^{\beta-1} \\
& =x^{\alpha-1}(1-x)^{\beta-1}[\alpha(1-x)-\beta x] \\
& =x^{\alpha-1}(1-x)^{\beta-1}[\alpha-(\alpha+\beta) x], \quad x \in(0,1) .
\end{aligned}
$$

This shows that $g_{\alpha, \beta}^{\prime}(x)>0$ for $x \in\left(0, \frac{\alpha}{\alpha+\beta}\right)$ and $g_{\alpha, \beta}^{\prime}(x)<0$ for $\left(\frac{\alpha}{\alpha+\beta}, 1\right)$, which proves the statement.

We need the following lemma that is of interest in itself:

Lemma 1 Let $\alpha, \beta>0$.

(i) If $0<\alpha+\beta \leq 1$, then $g_{\alpha, \beta}$ is strictly concave on $[0,1]$.

Define

$$
x_{1, \alpha, \beta}:=\frac{\alpha(\alpha+\beta-1)-\sqrt{\alpha \beta(\alpha+\beta-1)}}{(\alpha+\beta)(\alpha+\beta-1)}<\frac{\alpha}{\alpha+\beta}
$$

and

$$
x_{2, \alpha, \beta}:=\frac{\alpha(\alpha+\beta-1)+\sqrt{\alpha \beta(\alpha+\beta-1)}}{(\alpha+\beta)(\alpha+\beta-1)}>\frac{\alpha}{\alpha+\beta} .
$$

(ii) If $\alpha, \beta \in(0,1)$ with $\alpha+\beta>1$, then $g_{\alpha, \beta}$ is strictly concave on $[0,1]$.

(iii) If $\alpha>1$ and $\beta \in(0,1)$ then $g_{\alpha, \beta}$ is strictly convex on $\left(0, x_{1, \alpha, \beta}\right)$ and strictly concave on $\left(x_{1, \alpha, \beta}, 1\right)$.

(iv) If $\alpha \in(0,1)$ and $\beta>1$, then $g_{\alpha, \beta}$ is strictly concave on $\left(0, x_{2, \alpha, \beta}\right)$ and strictly convex on $\left(x_{2, \alpha, \beta}, 1\right)$.

(v) If $\alpha, \beta>1$, then $g_{\alpha, \beta}$ is strictly concave on $\left(x_{1, \alpha, \beta}, x_{2, \alpha, \beta}\right)$ and strictly convex on $\left(0, x_{1, \alpha, \beta}\right) \cup\left(x_{2, \alpha, \beta}, 1\right)$. 
Proof If we take the second derivative of $g_{\alpha, \beta}$ on $(0,1)$, then we get

$$
\begin{aligned}
g_{\alpha, \beta}^{\prime \prime}(x)= & \alpha(\alpha-1) x^{\alpha-2}(1-x)^{\beta}-\alpha \beta x^{\alpha-1}(1-x)^{\beta-1} \\
& -\alpha \beta x^{\alpha-1}(1-x)^{\beta-1}+\beta(\beta-1) x^{\alpha}(1-x)^{\beta-2} \\
= & \alpha(\alpha-1) x^{\alpha-2}(1-x)^{\beta}-2 \alpha \beta x^{\alpha-1}(1-x)^{\beta-1}+\beta(\beta-1) x^{\alpha}(1-x)^{\beta-2} \\
= & x^{\alpha-2}(1-x)^{\beta-2}\left[\alpha(\alpha-1)(1-x)^{2}-2 \alpha \beta x(1-x)+\beta(\beta-1) x^{2}\right]
\end{aligned}
$$

for all $\alpha, \beta>0$ and $x \in(0,1)$.

Now, consider the two-parameter family of parabolas

$$
h_{\alpha, \beta}(x):=\alpha(\alpha-1)(1-x)^{2}-2 \alpha \beta x(1-x)+\beta(\beta-1) x^{2}, \quad x \in \mathbb{R} .
$$

We have

$$
\begin{aligned}
h_{\alpha, \beta}(x) & =\alpha(\alpha-1)\left(x^{2}-2 x+1\right)-2 \alpha \beta\left(x-x^{2}\right)+\beta(\beta-1) x^{2} \\
& =[\alpha(\alpha-1)+2 \alpha \beta+\beta(\beta-1)] x^{2}-2(\alpha(\alpha-1)+\alpha \beta) x+\alpha(\alpha-1) \\
& =\left[\alpha^{2}+2 \alpha \beta+\beta^{2}-(\alpha+\beta)\right] x^{2}-2 \alpha(\alpha+\beta-1) x+\alpha(\alpha-1) \\
& =\left[(\alpha+\beta)^{2}-(\alpha+\beta)\right] x^{2}-2 \alpha(\alpha+\beta-1) x+\alpha(\alpha-1) \\
& =(\alpha+\beta)(\alpha+\beta-1) x^{2}-2 \alpha(\alpha+\beta-1) x+\alpha(\alpha-1)
\end{aligned}
$$

for $x \in \mathbb{R}$.

The discriminant of this family of parabolas is

$$
\begin{aligned}
\Delta_{\alpha, \beta} & :=4 \alpha^{2}(\alpha+\beta-1)^{2}-4(\alpha+\beta)(\alpha+\beta-1) \alpha(\alpha-1) \\
& =4 \alpha(\alpha+\beta-1)[\alpha(\alpha+\beta-1)-(\alpha+\beta)(\alpha-1)] \\
& =4 \alpha(\alpha+\beta-1)\left(\alpha^{2}+\alpha \beta-\alpha-\alpha^{2}-\alpha \beta+\alpha+\beta\right) \\
& =4 \alpha \beta(\alpha+\beta-1)
\end{aligned}
$$

for $\alpha, \beta>0$.

Now, if $0<\alpha+\beta<1$, then $\Delta_{\alpha, \beta}<0$ which shows that the parabola $h_{\alpha, \beta}(x)<0$ for all $x \in \mathbb{R}$, implying that $g_{\alpha, \beta}^{\prime \prime}(x)<0$ for $x \in(0,1)$, namely $g_{\alpha, \beta}$ is strictly concave on $[0,1]$.

If $\alpha+\beta=1$, then $h_{\alpha, \beta}(x)=\alpha(\alpha-1)<0$, namely $g_{\alpha, \beta}$ is strictly concave on $[0,1]$.

If $\alpha+\beta>1$ with $\alpha, \beta>0$ then $\Delta_{\alpha, \beta}>0$ and the parabola $h_{\alpha, \beta}(\cdot)$ has two distinct intercepts with the axis $o x$, namely

$$
x_{1, \alpha, \beta}=\frac{\alpha(\alpha+\beta-1)-\sqrt{\alpha \beta(\alpha+\beta-1)}}{(\alpha+\beta)(\alpha+\beta-1)}
$$

and

$$
x_{2, \alpha, \beta}=\frac{\alpha(\alpha+\beta-1)+\sqrt{\alpha \beta(\alpha+\beta-1)}}{(\alpha+\beta)(\alpha+\beta-1)} .
$$


The $x$ coordinate for the vertex is

$$
x_{V, \alpha, \beta}=\frac{\alpha}{\alpha+\beta} \in(0,1)
$$

for all $\alpha, \beta>0$.

We also have $h_{\alpha, \beta}(0)=\alpha(\alpha-1)$ and $h_{\alpha, \beta}(1)=\beta(\beta-1)$.

Now, if $\alpha, \beta \in(0,1)$ with $\alpha+\beta>1$ then $x_{1, \alpha, \beta}<0$ and $x_{2, \alpha, \beta}>1$, showing that $h_{\alpha, \beta}(x)<0$, namely $g_{\alpha, \beta}$ is strictly concave on $[0,1]$.

If $\alpha>1$ and $\beta \in(0,1)$, then $\alpha+\beta>1, x_{1, \alpha, \beta} \in\left(0, \frac{\alpha}{\alpha+\beta}\right), x_{2, \alpha, \beta}>1$, which shows that $h_{\alpha, \beta}(x)>0$ for $x \in\left(0, x_{1, \alpha, \beta}\right)$ and $h_{\alpha, \beta}(x)<0$ for $x \in\left(x_{1, \alpha, \beta}, 1\right)$, showing that $g_{\alpha, \beta}$ is strictly convex on $\left(0, x_{1, \alpha, \beta}\right)$ and strictly concave on $\left(x_{1, \alpha, \beta}, 1\right)$.

If $\alpha \in(0,1)$ and $\beta>1$, then $\alpha+\beta>1, x_{1, \alpha, \beta}<0, x_{2, \alpha, \beta} \in\left(\frac{\alpha}{\alpha+\beta}, 1\right)$, which shows that $h_{\alpha, \beta}(x)<0$ for $x \in\left(0, x_{2, \alpha, \beta}\right)$ and $h_{\alpha, \beta}(x)>0$ for $x \in\left(x_{2, \alpha, \beta}, 1\right)$, showing that $g_{\alpha, \beta}$ is strictly concave on $\left(0, x_{2, \alpha, \beta}\right)$ and strictly convex on $\left(x_{2, \alpha, \beta}, 1\right)$.

If $\alpha, \beta>1$, then $x_{1, \alpha, \beta} \in\left(0, \frac{\alpha}{\alpha+\beta}\right)$ and $x_{2, \alpha, \beta} \in\left(\frac{\alpha}{\alpha+\beta}, 1\right)$, which shows that $h_{\alpha, \beta}(x)<0$ for $\left(x_{1, \alpha, \beta}, x_{2, \alpha, \beta}\right)$ and $h_{\alpha, \beta}(x)>0$ for $x \in\left(0, x_{1, \alpha, \beta}\right) \cup\left(x_{2, \alpha, \beta}, 1\right)$ showing that $g_{\alpha, \beta}$ is strictly concave on $\left(x_{1, \alpha, \beta}, x_{2, \alpha, \beta}\right)$ and strictly convex on $\left(0, x_{1, \alpha, \beta}\right) \cup\left(x_{2, \alpha, \beta}, 1\right)$.

We can state the following fact concerning the logarithmic convexity of $f_{\alpha, \beta}$.

Proposition 2 Let $\alpha, \beta>0$. Define $x_{1, \alpha, \beta}$ and $x_{2, \alpha, \beta}$ as in Lemma 1 .

(1) If $\alpha, \beta \in(0,1)$, then $f_{\alpha, \beta}(x)$ is strictly log-concave on $[0,1]$.

(2) If $\alpha>1$ and $\beta \in(0,1)$ then $f_{\alpha, \beta}(x)$ is strictly log-convex on $\left(0, x_{1, \alpha, \beta}\right)$ and strictly log-concave on $\left(x_{1, \alpha, \beta}, 1\right)$.

(3) If $\alpha \in(0,1)$ and $\beta>1$, then $f_{\alpha, \beta}(x)$ is strictly log-concave on $\left(0, x_{2, \alpha, \beta}\right)$ and strictly log-convex on $\left(x_{2, \alpha, \beta}, 1\right)$.

(4) If $\alpha, \beta>1$, then $f_{\alpha, \beta}(x)$ is strictly log-concave on $\left(x_{1, \alpha, \beta}, x_{2, \alpha, \beta}\right)$ and strictly log-convex on $\left(0, x_{1, \alpha, \beta}\right) \cup\left(x_{2, \alpha, \beta}, 1\right)$.

The proof is obvious by Lemma 1 observing that $\ln \left[f_{\alpha, \beta}(x)\right]=g_{\alpha, \beta}(x)=x^{\alpha}(1-x)^{\beta}, x \in$ $[0,1]$ and $\alpha, \beta>0$.

We have also the following simple fact:

Proposition 3 Let $\alpha, \beta>0$. Then for all $x \in(0,1)$ we have the double inequality

$$
x^{\alpha}(1-x)^{\beta}<f_{\alpha, \beta}(x)-1<\frac{x^{\alpha}(1-x)^{\beta}}{1-x^{\alpha}(1-x)^{\beta}} .
$$

Proof We use the following elementary exponential inequality for $y \in(0,1)$, see [10]:

$$
y<e^{y}-1<\frac{y}{1-y}
$$

for $y \in(0,1)$. If in this inequality we take $g_{\alpha, \beta}(x)=y \in(0,1)$ for $x \in(0,1)$, we get the desired result (3.3). 


\section{Taylor's-type expansion for the generating function}

We have the following representation result:

Theorem 6 Let $\alpha, \beta>0$, then for all $x \in[0,1]$ and natural number $n \geq 1$, we have

$$
\begin{aligned}
f_{\alpha, \beta}(x)= & +\sum_{k=1}^{n} \frac{1}{k !} x^{\alpha k}(1-x)^{\beta k} \\
& +\frac{1}{n !} x^{\alpha(n+1)}(1-x)^{\beta(n+1)} \int_{0}^{1} \exp \left[s x^{\alpha}(1-x)^{\beta}\right](1-s)^{n} d s,
\end{aligned}
$$

where $x \in[0,1]$.

Proof Let $I \subset \mathbb{R}$ be a closed interval, $c \in I$ and let $n$ be a positive integer. If $f: I \longrightarrow \mathbb{C}$ is such that the $n$th derivative $f^{(n)}$ is absolutely continuous on $I$, then for each $y \in I$

$$
f(y)=T_{n}(f ; c, y)+R_{n}(f ; c, y),
$$

where $T_{n}(f ; c, y)$ is Taylor's polynomial, i.e.

$$
T_{n}(f ; c, y):=\sum_{k=0}^{n} \frac{(y-c)^{k}}{k !} f^{(k)}(c) .
$$

Note that $f^{(0)}:=f$ and $0 !:=1$, and the remainder is given by

$$
R_{n}(f ; c, y):=\frac{1}{n !} \int_{c}^{y}(y-t)^{n} f^{(n+1)}(t) d t .
$$

For any integrable function $h$ on an interval and any distinct numbers $c, d$ in that interval, we have, by the change of variable $t=(1-s) c+s d, s \in[0,1]$, that

$$
\int_{c}^{d} h(t) d t=(d-c) \int_{0}^{1} h((1-s) c+s d) d s
$$

Therefore,

$$
\begin{aligned}
& \int_{c}^{y} f^{(n+1)}(t)(y-t)^{n} d t \\
& \quad=(y-c) \int_{0}^{1} f^{(n+1)}((1-s) c+s y)(x-(1-s) c-s y)^{n} d s \\
& \quad=(y-c)^{n+1} \int_{0}^{1} f^{(n+1)}((1-s) c+s y)(1-s)^{n} d s,
\end{aligned}
$$

and from (4.4) we get the representation

$$
\begin{aligned}
f(y)= & \sum_{k=0}^{n} \frac{(y-c)^{k}}{k !} f^{(k)}(c) \\
& +\frac{1}{n !}(y-c)^{n+1} \int_{0}^{1} f^{(n+1)}((1-s) c+s y)(1-s)^{n} d s
\end{aligned}
$$

for all $y, c \in I$. 
Now, if we write equality (4.5) for the exponential function $f(y)=e^{y}, y \in \mathbb{R}$, and the point $c=0$, we get

$$
\exp y-1=\sum_{k=1}^{n} \frac{y^{k}}{k !}+\frac{1}{n !} y^{n+1} \int_{0}^{1} \exp (s y)(1-s)^{n} d s
$$

for any real number $y \in \mathbb{R}$.

If we take in (4.6) $y=g_{\alpha, \beta}(x), x \in[0,1]$, we get

$$
\begin{aligned}
\exp \left[g_{\alpha, \beta}(x)\right]-1= & \sum_{k=1}^{n} \frac{\left[g_{\alpha, \beta}(x)\right]^{k}}{k !} \\
& +\frac{1}{n !}\left[g_{\alpha, \beta}(x)\right]^{n+1} \int_{0}^{1} \exp \left[s g_{\alpha, \beta}(x)\right](1-s)^{n} d s,
\end{aligned}
$$

which produces the desired result (4.1).

We have some simple upper and lower bounds as follows:

Corollary 2 Let $\alpha, \beta>0$, then for all $x \in[0,1]$ and natural number $n \geq 1$, we have

$$
\begin{aligned}
& \sum_{k=1}^{n} \frac{1}{k !} x^{\alpha k}(1-x)^{\beta k} \\
& \quad \leq f_{\alpha, \beta}(x)-1 \\
& \quad \leq \sum_{k=1}^{n} \frac{1}{k !} x^{\alpha k}(1-x)^{\beta k}+\frac{e}{(n+1) !} x^{\alpha(n+1)}(1-x)^{\beta(n+1)}
\end{aligned}
$$

Proof The inequalities in (4.7) follow by (4.1) observing that

$$
\begin{aligned}
0 & \leq \frac{1}{n !}\left[g_{\alpha, \beta}(x)\right]^{n+1} \int_{0}^{1} \exp \left[s g_{\alpha, \beta}(x)\right](1-s)^{n} d s \\
& \leq \frac{1}{n !}\left[g_{\alpha, \beta}(x)\right]^{n+1} \max _{s \in[0,1]} \exp \left[s g_{\alpha, \beta}(x)\right] \int_{0}^{1}(1-s)^{n} d s \\
& \leq \frac{e}{(n+1) !}\left[g_{\alpha, \beta}(x)\right]^{n+1}
\end{aligned}
$$

for all $x \in[0,1]$.

Corollary 3 Let $\alpha, \beta>0$, then we have function series expansion

$$
f_{\alpha, \beta}(x)=1+\sum_{k=1}^{\infty} \frac{1}{k !} x^{\alpha k}(1-x)^{\beta k}
$$

uniformly on the interval $[0,1]$. 
Proof By (4.1) we have

$$
\begin{aligned}
& \left|f_{\alpha, \beta}(x)-1-\sum_{k=1}^{n} \frac{\left[g_{\alpha, \beta}(x)\right]^{k}}{k !}\right| \\
& \quad=\left|\frac{1}{n !}\left[g_{\alpha, \beta}(x)\right]^{n+1} \int_{0}^{1} \exp \left[s g_{\alpha, \beta}(x)\right](1-s)^{n} d s\right| \\
& \quad \leq \frac{1}{n !}\left[g_{\alpha, \beta}(x)\right]^{n+1} \int_{0}^{1}\left|\exp \left[s g_{\alpha, \beta}(x)\right](1-s)^{n}\right| d s \\
& \quad \leq \frac{e}{n !} \int_{0}^{1}(1-s)^{n} d s=\frac{e}{(n+1) !} \rightarrow 0
\end{aligned}
$$

uniformly for $x \in[0,1]$ as $n \rightarrow \infty$.

Now, we can introduce the two-variable function $F:(0, \infty) \times(0, \infty) \rightarrow(0, \infty)$, that we can call the Exponential-Beta function, defined by the integral

$$
F(\alpha, \beta):=\int_{0}^{1} \exp \left[x^{\alpha}(1-x)^{\beta}\right] d x>1
$$

Then we have the following representation result in terms of the beta function:

Theorem 7 For any natural number $n \geq 1$ and any $\alpha, \beta>0$, we have the representation

$$
F(\alpha, \beta)=1+\sum_{k=1}^{n} \frac{1}{k !} B(\alpha k+1, \beta k+1)+R_{n}(\alpha, \beta),
$$

where the remainder $R_{n}(\alpha, \beta)$ is given by

$$
\begin{aligned}
& R_{n}(\alpha, \beta) \\
& \quad:=\frac{1}{n !} \int_{0}^{1}\left(\int_{0}^{1}\left\{x^{\alpha(n+1)}(1-x)^{\beta(n+1)} \exp \left[s x^{\alpha}(1-x)^{\beta}\right]\right\} d x\right)(1-s)^{n} d s .
\end{aligned}
$$

Proof If we integrate identity (4.1), then we get

$$
\begin{aligned}
F(\alpha, \beta)-1= & \int_{0}^{1} f_{\alpha, \beta}(x) d x-1 \\
= & \sum_{k=1}^{n} \int_{0}^{1} \frac{1}{k !}\left[g_{\alpha, \beta}(x)\right]^{k} d x \\
& +\frac{1}{n !} \int_{0}^{1}\left[g_{\alpha, \beta}(x)\right]^{n+1}\left(\int_{0}^{1} \exp \left[\operatorname{sg} g_{\alpha, \beta}(x)\right](1-s)^{n} d s\right) d x \\
= & \sum_{k=1}^{n} \int_{0}^{1} \frac{\left[x^{\alpha}(1-x)^{\beta}\right]^{k}}{k !} d x \\
& +\frac{1}{n !} \int_{0}^{1}\left[x^{\alpha}(1-x)^{\beta}\right]^{n+1}\left(\int_{0}^{1} \exp \left[s x^{\alpha}(1-x)^{\beta}\right](1-s)^{n} d s\right) d x \\
= & \sum_{k=1}^{n} \frac{1}{k !} \int_{0}^{1} x^{\alpha k}(1-x)^{\beta k} d x
\end{aligned}
$$




$$
\begin{aligned}
& +\frac{1}{n !} \int_{0}^{1} x^{\alpha(n+1)}(1-x)^{\beta(n+1)}\left(\int_{0}^{1} \exp \left[s x^{\alpha}(1-x)^{\beta}\right](1-s)^{n} d s\right) d x \\
= & \sum_{k=1}^{n} \frac{1}{k !} B(\alpha k+1, \beta k+1) \\
& +\frac{1}{n !} \int_{0}^{1}\left(\int_{0}^{1}\left\{x^{\alpha(n+1)}(1-x)^{\beta(n+1)} \exp \left[s x^{\alpha}(1-x)^{\beta}\right]\right\} d x\right)(1-s)^{n} d s,
\end{aligned}
$$

where for the last equality we used Fubini's theorem.

Corollary 4 We have the following beta series expansion:

$$
F(\alpha, \beta)=1+\sum_{k=1}^{\infty} \frac{1}{k !} B(\alpha k+1, \beta k+1)
$$

uniformly over $\alpha, \beta>0$.

Proof We observe that for all $\alpha, \beta>0$ we have

$$
\left|R_{n}(\alpha, \beta)\right| \leq \frac{e}{n !} \int_{0}^{1}(1-s)^{n} d s=\frac{e}{(n+1) !} \rightarrow 0
$$

as $n \rightarrow \infty$, which proves the claim.

Theorem 8 For any natural number $n \geq 1$ and any $\alpha, \beta>0$, we have the representation

$$
\ln \left[f_{\alpha, \beta}(x)\right]=x^{\alpha}+\sum_{k=1}^{n}(-1)^{k} \frac{\beta(\beta-1) \cdots(\beta-k+1)}{k !} x^{k+\alpha}+E_{n}(\alpha, \beta)
$$

where the remainder $E_{n}(\alpha, \beta)$ is given by

$$
E_{n}(\alpha, \beta):=(-1)^{n+1} \frac{\beta(\beta-1) \cdots(\beta-n)}{n !} x^{n+\alpha+1} \int_{0}^{1}(1-s x)^{\beta-n-1}(1-s)^{n} d s .
$$

Proof Consider the function $f:(-1,1) \rightarrow(0, \infty), f(x)=(1-x)^{\beta}$. Then

$$
f^{(k)}(0)=(-1)^{k} \beta(\beta-1) \cdots(\beta-k+1) \quad \text { for } k=1, \ldots, n,
$$

and

$$
f^{(n+1)}(x)=(-1)^{n+1} \beta(\beta-1) \cdots(\beta-n)(1-x)^{\beta-n-1} .
$$

Using the representation (4.5) for $c=0$, we get

$$
\begin{aligned}
(1-x)^{\beta}=1 & +\sum_{k=1}^{n}(-1)^{k} \frac{\beta(\beta-1) \cdots(\beta-k+1)}{k !} x^{k} \\
& +(-1)^{n+1} \frac{\beta(\beta-1) \cdots(\beta-n)}{n !} x^{n+1} \int_{0}^{1}(1-s x)^{\beta-n-1}(1-s)^{n} d s
\end{aligned}
$$

for all $x \in(0,1)$. 
If we multiply this equality by $x^{\alpha}$, we get

$$
\begin{aligned}
x^{\alpha}(1-x)^{\beta}= & x^{\alpha}+\sum_{k=1}^{n}(-1)^{k} \frac{\beta(\beta-1) \cdots(\beta-k+1)}{k !} x^{k+\alpha} \\
& +(-1)^{n+1} \frac{\beta(\beta-1) \cdots(\beta-n)}{n !} x^{n+\alpha+1} \int_{0}^{1}(1-s x)^{\beta-n-1}(1-s)^{n} d s,
\end{aligned}
$$

which is equivalent to the desired representation (4.11).

Remark 1 If we take the exponential in (4.11), then we get

$$
\begin{aligned}
& f_{\alpha, \beta}(x) \\
& \quad=\exp \left[x^{\alpha}+\sum_{k=1}^{n}(-1)^{k} \frac{\beta(\beta-1) \cdots(\beta-k+1)}{k !} x^{k+\alpha}+E_{n}(\alpha, \beta)\right] \\
& =e^{x^{\alpha}} \prod_{k=1}^{n} \exp \left[(-1)^{k} \frac{\beta(\beta-1) \cdots(\beta-k+1)}{k !} x^{k+\alpha}\right] e^{E_{n}(\alpha, \beta)}
\end{aligned}
$$

for $x \in(0,1)$ and $\alpha, \beta>0$.

\section{Some analytic inequalities}

We start with the following fact:

Theorem 9 Let $\alpha, \beta>0$. For any $p, q>1$ with $\frac{1}{p}+\frac{1}{q}=1$, we have

$$
0 \leq f_{\alpha, \beta}(x)-1 \leq\left[\exp \left(x^{\alpha p}\right)-1\right]^{1 / p}\left[\exp \left((1-x)^{q \beta}\right)-1\right]^{1 / q}
$$

for all $x \in[0,1]$.

In particular, we have

$$
\left[f_{\alpha, \beta}(x)-1\right]^{2} \leq\left[\exp \left(x^{2 \alpha}\right)-1\right]\left[\exp \left((1-x)^{2 \beta}\right)-1\right]
$$

for all $x \in[0,1]$.

Proof If we make use of Hölder's discrete weighted inequality

$$
0 \leq \sum_{k=1}^{n} m_{k} a_{k} b_{k} \leq\left(\sum_{k=1}^{n} m_{k} a_{k}^{p}\right)^{1 / p}\left(\sum_{k=1}^{n} m_{k} b_{k}^{q}\right)^{1 / q}
$$

where $m_{k}, a_{k}, b_{k} \geq 0, k \in\{1, \ldots, n\}$ and $p, q>1$ with $\frac{1}{p}+\frac{1}{q}=1$, then we can write

$$
\begin{aligned}
0 & \leq \sum_{k=1}^{n} \frac{1}{k !} x^{\alpha k}(1-x)^{\beta k} \leq\left(\sum_{k=1}^{n} \frac{1}{k !} x^{\alpha p k}\right)^{1 / p}\left(\sum_{k=1}^{n} \frac{1}{k !}(1-x)^{q \beta k}\right)^{1 / q} \\
& =\left(\sum_{k=1}^{n} \frac{1}{k !}\left(x^{\alpha p}\right)^{k}\right)^{1 / p}\left(\sum_{k=1}^{n} \frac{1}{k !}\left[(1-x)^{q \beta}\right]^{k}\right)^{1 / q}
\end{aligned}
$$

for all $n>1$ and $x \in[0,1]$. 
Since the series $\sum_{k=1}^{\infty} \frac{1}{k !} x^{\alpha k}(1-x)^{\beta k}, \sum_{k=1}^{\infty} \frac{1}{k !}\left(x^{\alpha p}\right)^{k}$ and $\sum_{k=1}^{\infty} \frac{1}{k !}\left[(1-x)^{q \beta}\right]^{k}$ are convergent and

$$
\begin{aligned}
& \sum_{k=1}^{\infty} \frac{1}{k !} x^{\alpha k}(1-x)^{\beta k}=\exp \left[x^{\alpha}(1-x)^{\beta}\right]-1=f_{\alpha, \beta}(x)-1, \\
& \sum_{k=1}^{\infty} \frac{1}{k !}\left(x^{\alpha p}\right)^{k}=\exp \left(x^{\alpha p}\right)-1
\end{aligned}
$$

and

$$
\sum_{k=1}^{\infty} \frac{1}{k !}\left[(1-x)^{q \beta}\right]^{k}=\exp \left[(1-x)^{q \beta}\right]-1
$$

then, by taking the limit as $n \rightarrow \infty$ in (5.3), we get the desired result (5.1).

The following result also provides some lower bounds for $f_{\alpha, \beta}(x)$.

Theorem 10 Let $\alpha, \beta>0$. Then for all $x \in[0,1]$, we have

$$
\begin{aligned}
& \frac{1}{e-1}\left[\exp \left(x^{\alpha}\right)-1\right]\left[\exp \left((1-x)^{\beta}\right)-1\right] \\
& \quad \leq f_{\alpha, \beta}(x)-1 \\
& \quad \leq \frac{1}{e-1}\left[\exp \left(x^{\alpha}\right)-1\right]\left[\exp \left((1-x)^{\beta}\right)-1\right]+\frac{1}{4}(e-1) x^{\alpha}(1-x)^{\beta} .
\end{aligned}
$$

Proof We use the weighted Čebyšev's inequality for sequences $a_{k}, b_{k}, k \in\{1, \ldots, n\}$ that have the same monotonicity

$$
\sum_{k=1}^{n} m_{k} a_{k} \sum_{k=1}^{n} m_{k} b_{k} \leq \sum_{k=1}^{n} m_{k} \sum_{k=1}^{n} m_{k} a_{k} b_{k}
$$

where $m_{k} \geq 0, k \in\{1, \ldots, n\}$.

Consider the sequences $a_{k}:=x^{\alpha k}, b_{k}:=(1-x)^{\beta k}, k \in\{1, \ldots, n\}$, for $x \in[0,1]$. We observe that both sequences are monotonic nonincreasing and, by applying Čebyšev's inequality for the positive weights $m_{k}:=\frac{1}{k !}$, we get

$$
\sum_{k=1}^{n} \frac{1}{k !} x^{\alpha k} \sum_{k=1}^{n} \frac{1}{k !}(1-x)^{\beta k} \leq \sum_{k=1}^{n} \frac{1}{k !} \sum_{k=1}^{n} \frac{1}{k !} x^{\alpha k}(1-x)^{\beta k}
$$

for all $x \in[0,1]$ and $n \geq 1$.

Since the series $\sum_{k=1}^{\infty} \frac{1}{k !} x^{\alpha k}, \sum_{k=1}^{\infty} \frac{1}{k !}(1-x)^{\beta k}$ and $\sum_{k=1}^{\infty} \frac{1}{k !}$ are convergent and

$$
\begin{aligned}
& \sum_{k=1}^{\infty} \frac{1}{k !} x^{\alpha k}=\sum_{k=1}^{\infty} \frac{1}{k !}\left(x^{\alpha}\right)^{k}=\exp \left(x^{\alpha}\right)-1 \\
& \sum_{k=1}^{\infty} \frac{1}{k !}(1-x)^{\beta k}=\sum_{k=1}^{\infty} \frac{1}{k !}\left((1-x)^{\beta}\right)^{k}=\exp \left((1-x)^{\beta}\right)-1
\end{aligned}
$$


and $\sum_{k=1}^{\infty} \frac{1}{k !}=e-1$, then, by taking the limit as $n \rightarrow \infty$ in (5.6), we get the first inequality in (5.4).

Further, we use the weighted Grüss inequality for the bounded sequences $a \leq a_{k} \leq A$, $b \leq b_{k} \leq B, k \in\{1, \ldots, n\}$ and nonnegative weights $m_{k} \geq 0, k \in\{1, \ldots, n\}$,

$$
\begin{gathered}
\left|\sum_{k=1}^{n} m_{k} \sum_{k=1}^{n} m_{k} a_{k} b_{k}-\sum_{k=1}^{n} m_{k} a_{k} \sum_{k=1}^{n} m_{k} b_{k}\right| \\
\leq \frac{1}{4}\left(\sum_{k=1}^{n} m_{k}\right)^{2}(A-a)(B-b) .
\end{gathered}
$$

Now, if we consider the sequences $a_{k}:=x^{\alpha k}, b_{k}:=(1-x)^{\beta k} k \in\{1, \ldots, n\}$, for $x \in[0,1]$, then we observe that $0 \leq a_{k} \leq x^{\alpha}$ and $0 \leq b_{k} \leq(1-x)^{\beta}$ for all natural numbers $k \geq 1$. So, by utilising inequality (5.7) for $a=b=0, A=x^{\alpha}, B=(1-x)^{\beta}$ and $m_{k}:=\frac{1}{k !}$, we get

$$
\begin{aligned}
& \left|\sum_{k=1}^{n} \frac{1}{k !} \sum_{k=1}^{n} \frac{1}{k !} x^{\alpha k}(1-x)^{\beta k}-\sum_{k=1}^{n} \frac{1}{k !} x^{\alpha k} \sum_{k=1}^{n} \frac{1}{k !}(1-x)^{\beta k}\right| \\
& \quad \leq \frac{1}{4}\left(\sum_{k=1}^{n} \frac{1}{k !}\right)^{2} x^{\alpha}(1-x)^{\beta},
\end{aligned}
$$

which holds for all $x \in[0,1]$ and $n \geq 1$.

Since all the series are involved in (5.8), by taking the limit as $n \rightarrow \infty$ in this inequality, we get the second part of (5.4).

Theorem 11 Let $\alpha, \beta>0$. Then for all $x \in(0,1)$, we have

$$
(e-1)\left[x^{\alpha}(1-x)^{\beta}\right]^{\frac{e}{e-1}} \leq f_{\alpha, \beta}(x)-1
$$

and

$$
\begin{aligned}
0 & \leq \ln \left(\frac{f_{\alpha, \beta}(x)-1}{e-1}\right)-\ln \left\{\left[x^{\alpha}(1-x)^{\beta}\right]^{\frac{e}{e-1}}\right\} \\
& \leq \frac{1}{(e-1)^{2}}\left[f_{-\alpha,-\beta}(x)-1\right]\left[f_{\alpha, \beta}(x)-1\right]-1 .
\end{aligned}
$$

Proof Since $\ln$ is a concave function, by Jensen's discrete inequality for concave functions $g$, namely

$$
g\left(\frac{\sum_{k=1}^{n} p_{k} x_{k}}{\sum_{k=1}^{n} p_{k}}\right) \geq \frac{\sum_{k=1}^{n} p_{k} g\left(x_{k}\right)}{\sum_{k=1}^{n} p_{k}}
$$

where $p_{k}>0, k \in\{1, \ldots, n\}$, we have for $x_{k}=x^{\alpha k}(1-x)^{\beta k}$ and $p_{k}=\frac{1}{k !}, k \in\{1, \ldots, n\}$ that

$$
\begin{aligned}
\ln \left(\frac{\sum_{k=1}^{n} \frac{1}{k !} x^{\alpha k}(1-x)^{\beta k}}{\sum_{k=1}^{n} \frac{1}{k !}}\right) & \geq \frac{\sum_{k=1}^{n} \frac{1}{k !} \ln \left[x^{\alpha k}(1-x)^{\beta k}\right]}{\sum_{k=1}^{n} \frac{1}{k !}} \\
& =\frac{\sum_{k=1}^{n} \frac{k}{k !} \ln \left[x^{\alpha}(1-x)^{\beta}\right]}{\sum_{k=1}^{n} \frac{1}{k !}}
\end{aligned}
$$




$$
\begin{aligned}
& =\ln \left[x^{\alpha}(1-x)^{\beta}\right] \frac{\sum_{k=1}^{n} \frac{k}{k !}}{\sum_{k=1}^{n} \frac{1}{k !}} \\
& =\ln \left[x^{\alpha}(1-x)^{\beta}\right] \frac{\sum_{k=1}^{n} \frac{1}{(k-1) !}}{\sum_{k=1}^{n} \frac{1}{k !}}
\end{aligned}
$$

for all $x \in(0,1)$ and $n \geq 1$.

Since $\sum_{k=1}^{\infty} \frac{1}{(k-1) !}=e$ and $\sum_{k=1}^{\infty} \frac{1}{k !}=e-1$, by taking the limit as $n \rightarrow \infty$ in (5.11), we get

$$
\ln \left(\frac{f_{\alpha, \beta}(x)-1}{e-1}\right) \geq \frac{e}{e-1} \ln \left[x^{\alpha}(1-x)^{\beta}\right]=\ln \left\{\left[x^{\alpha}(1-x)^{\beta}\right]^{\frac{e}{e-1}}\right\},
$$

which is equivalent to the first inequality in (5.9).

Further, we use Dragomir-Ionescu's reverse of Jensen's inequality [6] for concave functions

$$
\begin{aligned}
0 & \leq g\left(\frac{\sum_{k=1}^{n} p_{k} x_{k}}{\sum_{k=1}^{n} p_{k}}\right)-\frac{\sum_{k=1}^{n} p_{k} g\left(x_{k}\right)}{\sum_{k=1}^{n} p_{k}} \\
& \leq \frac{1}{\sum_{k=1}^{n} p_{k}} \sum_{k=1}^{n} p_{k} g^{\prime}\left(x_{k}\right) \frac{1}{\sum_{k=1}^{n} p_{k}} \sum_{k=1}^{n} p_{k} x_{k}-\frac{1}{\sum_{k=1}^{n} p_{k}} \sum_{k=1}^{n} p_{k} x_{k} g^{\prime}\left(x_{k}\right),
\end{aligned}
$$

which gives for $g(x)=\ln x, x_{k}=x^{\alpha k}(1-x)^{\beta k}$ and $p_{k}=\frac{1}{k !}, k \in\{1, \ldots, n\}$ that

$$
\begin{aligned}
0 & \leq \ln \left(\frac{\sum_{k=1}^{n} \frac{1}{k !} x^{\alpha k}(1-x)^{\beta k}}{\sum_{k=1}^{n} \frac{1}{k !}}\right)-\frac{\sum_{k=1}^{n} \frac{1}{k !} \ln \left[x^{\alpha k}(1-x)^{\beta k}\right]}{\sum_{k=1}^{n} \frac{1}{k !}} \\
& \leq \frac{1}{\sum_{k=1}^{n} \frac{1}{k !}} \sum_{k=1}^{n} \frac{1}{k !} x^{-\alpha k}(1-x)^{-\beta k} \frac{1}{\sum_{k=1}^{n} \frac{1}{k !} \sum_{k=1}^{n} \frac{1}{k !} x^{\alpha k}(1-x)^{\beta k}-1}
\end{aligned}
$$

for $x \in(0,1)$ and $n \geq 1$.

Since the series $\sum_{k=1}^{\infty} \frac{1}{k !} x^{-\alpha k}(1-x)^{-\beta k}$ is convergent and

$$
\sum_{k=1}^{\infty} \frac{1}{k !} x^{-\alpha k}(1-x)^{-\beta k}=\sum_{k=1}^{\infty} \frac{1}{k !}\left[x^{-\alpha}(1-x)^{-\beta}\right]^{k}=\exp \left[x^{-\alpha}(1-x)^{-\beta}\right]-1,
$$

by letting $n \rightarrow \infty$ in (5.13), we get

$$
\begin{aligned}
0 & \leq \ln \left(\frac{f_{\alpha, \beta}(x)-1}{e-1}\right)-\ln \left\{\left[x^{\alpha}(1-x)^{\beta}\right]^{\frac{e}{e-1}}\right\} \\
& \leq \frac{1}{(e-1)^{2}}\left\{\exp \left[x^{-\alpha}(1-x)^{-\beta}\right]-1\right\}\left\{\exp \left[x^{\alpha}(1-x)^{\beta}\right]-1\right\}-1,
\end{aligned}
$$

which is equivalent to (5.10).

Remark 2 As a simple consequence of inequality (5.10) we note that

$$
(e-1)^{2} \leq\left[f_{-\alpha,-\beta}(x)-1\right]\left[f_{\alpha, \beta}(x)-1\right]
$$

for all positive $\alpha, \beta>0$ and $x \in(0,1)$. 
In 1984, S.S. Dragomir [3] obtained the following Cauchy-Bunyakovsky-Schwarz related weighted inequality (see also [4, Theorem 2.20]):

$$
\sum_{k=1}^{n} m_{k} a_{k}^{2} \sum_{k=1}^{n} m_{k} b_{k}^{2} \geq \frac{\sum_{k=1}^{n} m_{k} a_{k} \sum_{k=1}^{n} m_{k} b_{k} \sum_{k=1}^{n} m_{k} a_{k} b_{k}}{\sum_{k=1}^{n} m_{k}}
$$

where $a_{k}, b_{k}$ are real numbers, $m_{k} \geq 0$ for $k \in\{1, \ldots, n\}$, and $\sum_{k=1}^{n} m_{k}>0$.

Theorem 12 Let $\alpha, \beta>0$. Then for all $x \in(0,1)$ we have

$$
0 \leq f_{\alpha, \beta}(x)-1 \leq(e-1) \frac{\left[\exp \left(x^{2 \alpha}\right)-1\right]\left[\exp \left((1-x)^{2 \beta}\right)-1\right]}{\left[\exp \left(x^{\alpha}\right)-1\right]\left[\exp \left((1-x)^{\beta}\right)-1\right]}
$$

Proof By taking $a_{k}:=x^{\alpha k}, b_{k}:=(1-x)^{\beta k}$ and $m_{k}:=\frac{1}{k !}$ in (5.15), we get

$$
\begin{aligned}
& \sum_{k=1}^{n} \frac{1}{k !} x^{2 \alpha k} \sum_{k=1}^{n} \frac{1}{k !}(1-x)^{2 \beta k} \\
& \quad \geq \frac{\sum_{k=1}^{n} \frac{1}{k !} x^{\alpha k} \sum_{k=1}^{n} \frac{1}{k !}(1-x)^{\beta k} \sum_{k=1}^{n} \frac{1}{k !} x^{\alpha k}(1-x)^{\beta k}}{\sum_{k=1}^{n} \frac{1}{k !}} .
\end{aligned}
$$

Since all the series involved in (5.17) are convergent, by taking the limit as $n \rightarrow \infty$ in this inequality, we get the desired result (5.16).

Now, recall the well-known inequality between the weighted arithmetic mean and weighted geometric mean,

$$
a^{1-t} b^{t} \leq(1-t) a+t b
$$

which holds for all $a, b>0$ and $t \in[0,1]$. This inequality is also known in the literature as Young's inequality.

We have the following global convexity result for the function $f_{\alpha, \beta}(x)$ as a mapping of the positive parameters $(\alpha, \beta)$. More precisely, we have

\section{Theorem 13 The mapping}

$$
(0, \infty) \times(0, \infty) \ni(\alpha, \beta) \quad \mapsto \quad f_{\alpha, \beta}(x) \in[0, \infty)
$$

is globally convex on $(0, \infty) \times(0, \infty)$ for any $x \in(0,1)$.

Proof Fix $x \in(0,1)$. Let $\left(\alpha_{1}, \beta_{1}\right),\left(\alpha_{2}, \beta_{2}\right) \in(0, \infty) \times(0, \infty)$ and $t \in[0,1]$. Then

$$
(1-t)\left(\alpha_{1}, \beta_{1}\right)+t\left(\alpha_{2}, \beta_{2}\right)=\left((1-t) \alpha_{1}+t \alpha_{2},(1-t) \beta_{1}+t \beta_{2}\right) \in(0, \infty) \times(0, \infty)
$$


and

$$
\begin{aligned}
f_{(1-t) \alpha_{1}+t \alpha_{2},(1-t) \beta_{1}+t \beta_{2}}(x)-1 & =\sum_{k=1}^{\infty} \frac{1}{k !} x^{\left[(1-t) \alpha_{1}+t \alpha_{2}\right] k}(1-x)^{\left[(1-t) \beta_{1}+t \beta_{2}\right] k} \\
& =\sum_{k=1}^{\infty} \frac{1}{k !} x^{(1-t) \alpha_{1} k+t \alpha_{2} k}(1-x)^{k(1-t) \beta_{1}+t \beta_{2} k} \\
& =\sum_{k=1}^{\infty} \frac{1}{k !} x^{(1-t) \alpha_{1} k} x^{t \alpha_{2} k}(1-x)^{k(1-t) \beta_{1}}(1-x)^{t \beta_{2} k} \\
& =\sum_{k=1}^{\infty} \frac{1}{k !} x^{(1-t) \alpha_{1} k}(1-x)^{k(1-t) \beta_{1}} x^{t \alpha_{2} k}(1-x)^{t \beta_{2} k} \\
& =\sum_{k=1}^{\infty} \frac{1}{k !}\left[x^{\alpha_{1} k}(1-x)^{k \beta_{1}}\right]^{(1-t)}\left[x^{\alpha_{2} k}(1-x)^{\beta_{2} k}\right]^{t}=: A .
\end{aligned}
$$

By Young's inequality (GA), we have

$$
\begin{aligned}
& {\left[x^{\alpha_{1} k}(1-x)^{k \beta_{1}}\right]^{(1-t)}\left[x^{\alpha_{2} k}(1-x)^{\beta_{2} k}\right]^{t}} \\
& \quad \leq(1-t) x^{\alpha_{1} k}(1-x)^{k \beta_{1}}+t x^{\alpha_{2} k}(1-x)^{\beta_{2} k} \\
& \quad=(1-t)\left[x^{\alpha_{1}}(1-x)^{\beta_{1}}\right]^{k}+t\left[x^{\alpha_{2}}(1-x)^{\beta_{2}}\right]^{k}
\end{aligned}
$$

for all $k \geq 1$, and, by summing this inequality over $k$, we get

$$
\begin{aligned}
A & \leq \sum_{k=1}^{\infty} \frac{1}{k !}\left\{(1-t)\left[x^{\alpha_{1}}(1-x)^{\beta_{1}}\right]^{k}+t\left[x^{\alpha_{2}}(1-x)^{\beta_{2}}\right]^{k}\right\} \\
& =(1-t) \sum_{k=1}^{\infty} \frac{1}{k !}\left[x^{\alpha_{1}}(1-x)^{\beta_{1}}\right]^{k}+t \sum_{k=1}^{\infty} \frac{1}{k !}\left[x^{\alpha_{2}}(1-x)^{\beta_{2}}\right]^{k} \\
& =(1-t)\left[f_{\left(\alpha_{1}, \beta_{1}\right)}(x)-1\right]+t\left[f_{\left(\alpha_{2}, \beta_{2}\right)}(x)-1\right] \\
& =(1-t) f_{\left(\alpha_{1}, \beta_{1}\right)}(x)+t f_{\left(\alpha_{2}, \beta_{2}\right)}(x)-1,
\end{aligned}
$$

which implies that

$$
f_{(1-t) \alpha_{1}+t \alpha_{2},(1-t) \beta_{1}+t \beta_{2}}(x) \leq(1-t) f_{\left(\alpha_{1}, \beta_{1}\right)}(x)+t f_{\left(\alpha_{2}, \beta_{2}\right)}(x)
$$

and the claim is thus proved.

Corollary 5 The function $F:(0, \infty) \times(0, \infty) \rightarrow[0, \infty)$ defined by

$$
F(\alpha, \beta)=\int_{0}^{1} f_{\alpha, \beta}(x) d x
$$

is convex as a function of two variables on $(0, \infty) \times(0, \infty)$. 
Proof Let $\left(\alpha_{1}, \beta_{1}\right),\left(\alpha_{2}, \beta_{2}\right) \in(0, \infty) \times(0, \infty)$ and $t \in[0,1]$. Then

$$
\begin{aligned}
F\left((1-t) \alpha_{1}+t \alpha_{2},(1-t) \beta_{1}+t \beta_{2}\right) & =\int_{0}^{1} f_{(1-t) \alpha_{1}+t \alpha_{2},(1-t) \beta_{1}+t \beta_{2}}(x) d x \\
& \leq \int_{0}^{1}\left[(1-t) f_{\left(\alpha_{1}, \beta_{1}\right)}(x)+t f_{\left(\alpha_{2}, \beta_{2}\right)}(x)\right] d x \\
& =(1-t) \int_{0}^{1} f_{\left(\alpha_{1}, \beta_{1}\right)}(x) d x+t \int_{0}^{1} f_{\left(\alpha_{2}, \beta_{2}\right)}(x) d x \\
& =(1-t) F\left(\alpha_{1}, \beta_{1}\right)+t F\left(\alpha_{2}, \beta_{2}\right),
\end{aligned}
$$

which proves the statement.

Finally, we have the following logarithmic convexity property:

Theorem 14 The function $F-1$ is logarithmically convex on $(0, \infty) \times(0, \infty)$ as a function of two variables.

Proof Let $\left(\alpha_{1}, \beta_{1}\right),\left(\alpha_{2}, \beta_{2}\right) \in(0, \infty) \times(0, \infty)$ and $t \in[0,1]$. Then by the representation (4.10) we have

$$
\begin{aligned}
F & \left((1-t) \alpha_{1}+t \alpha_{2},(1-t) \beta_{1}+t \beta_{2}\right)-1 \\
& =\sum_{k=1}^{\infty} \frac{1}{k !} B\left(\left[(1-t) \alpha_{1}+t \alpha_{2}\right] k+1,\left[(1-t) \beta_{1}+t \beta_{2}\right] k+1\right) \\
& =\sum_{k=1}^{\infty} \frac{1}{k !} B(1-t)\left(\alpha_{1} k+1\right)+t\left(\alpha_{2} k+1\right),(1-t)\left(\beta_{1} k+1\right)+t\left(\beta_{2} k+1\right) \\
& =\sum_{k=1}^{\infty} \frac{1}{k !} B\left[(1-t)\left(\alpha_{1} k+1, \beta_{1} k+1\right)+t\left(\alpha_{2} k+1, \beta_{2} k+1\right)\right]=: T .
\end{aligned}
$$

By the logarithmic convexity of the beta function, we have

$$
\begin{aligned}
& B\left[(1-t)\left(\alpha_{1} k+1, \beta_{1} k+1\right)+t\left(\alpha_{2} k+1, \beta_{2} k+1\right)\right] \\
& \quad \leq\left[B\left(\alpha_{1} k+1, \beta_{1} k+1\right)\right]^{1-t}\left[B\left(\alpha_{2} k+1, \beta_{2} k+1\right)\right]^{t}
\end{aligned}
$$

for $\left(\alpha_{1}, \beta_{1}\right),\left(\alpha_{2}, \beta_{2}\right) \in(0, \infty) \times(0, \infty)$ and $t \in[0,1]$.

This implies that

$$
\begin{aligned}
T & \leq \sum_{k=1}^{\infty} \frac{1}{k !}\left[B\left(\alpha_{1} k+1, \beta_{1} k+1\right)\right]^{1-t}\left[B\left(\alpha_{2} k+1, \beta_{2} k+1\right)\right]^{t} \\
& \leq \sum_{k=1}^{\infty} \frac{1}{k !}\left[B\left(\alpha_{1} k+1, \beta_{1} k+1\right)\right]^{1-t}\left[B\left(\alpha_{2} k+1, \beta_{2} k+1\right)\right]^{t} \\
& \leq\left[\sum_{k=1}^{\infty} \frac{1}{k !}\left(\left[B\left(\alpha_{1} k+1, \beta_{1} k+1\right)\right]^{1-t}\right)^{\frac{1}{1-t}}\right]^{1-t}
\end{aligned}
$$




$$
\begin{gathered}
\quad \times\left[\sum_{k=1}^{\infty} \frac{1}{k !}\left(\left[B\left(\alpha_{2} k+1, \beta_{2} k+1\right)\right]^{t}\right)^{\frac{1}{t}}\right]^{t} \\
=\left[\sum_{k=1}^{\infty} \frac{1}{k !}\left[B\left(\alpha_{1} k+1, \beta_{1} k+1\right)\right]\right]^{1-t} \\
\times\left[\sum_{k=1}^{\infty} \frac{1}{k !}\left[B\left(\alpha_{2} k+1, \beta_{2} k+1\right)\right]\right]^{t},
\end{gathered}
$$

where for the last inequality we used Hölder's inequality with $p=\frac{1}{1-t}$ and $q=\frac{1}{t}$, for which we have $\frac{1}{p}+\frac{1}{q}=1$ with $p, q>1$.

Therefore, we have

$$
\begin{aligned}
& F\left((1-t) \alpha_{1}+t \alpha_{2},(1-t) \beta_{1}+t \beta_{2}\right)-1 \\
& \quad \leq\left[F\left(\alpha_{1}, \beta_{1}\right)-1\right]^{1-t}\left[F\left(\alpha_{2}, \beta_{2}\right)-1\right]^{t}
\end{aligned}
$$

for $\left(\alpha_{1}, \beta_{1}\right),\left(\alpha_{2}, \beta_{2}\right) \in(0, \infty) \times(0, \infty)$ and $t \in[0,1]$.

This proves the statement.

\section{Acknowledgements}

The authors are thankful to the editor and anonymous referees for their valuable comments and suggestions.

\section{Funding}

There is no funding for this research article.

\section{Availability of data and materials}

Not applicable.

\section{Competing interests}

The authors declare that they have no competing interests.

\section{Authors' contributions}

The authors contributed equally to the writing of this paper. The authors read and approved the final manuscript.

\section{Publisher's Note}

Springer Nature remains neutral with regard to jurisdictional claims in published maps and institutional affiliations.

Received: 24 July 2019 Accepted: 23 September 2019 Published online: 01 October 2019

\section{References}

1. Alzer, H., Paris, R.B.: A mean value inequality for Euler's beta function. Ir. Math. Soc. Bull. 78, 25-30 (2016)

2. Andrews, L.C.: Special Functions for Engineers and Applied Mathematicians. Macmillan Co., New York (1985)

3. Dragomir, S.S.: On some inequalities (Romanian). In: Caiete Metodico-Ştiinţifice, Romania, vol. 13, p. 20 (1984) Faculty of Mathematics, Timişoara University

4. Dragomir, S.S.: A survey on Cauchy-Bunyakovsky-Schwarz discrete inequality. J. Inequal. Pure Appl. Math. 4(3), Article ID 63 (2003)

5. Dragomir, S.S., Agarwal, R.P., Barnett, N.S.: Inequalities for beta and gamma functions via some classical and new integral inequalities. J. Inequal. Appl. 5(2), 103-165 (2000)

6. Dragomir, S.S., Ionescu, N.M.: Some converse of Jensen's inequality and applications. Rev. Anal. Numér. Théor. Approx. $23,71-78(1994)$

7. Khosrowshahi, F.: Value profile analysis of construction projects. J. Financ. Manag. Prop. Constr. 1(1), 55-77 (1996)

8. Khosrowshahi, F., Kaka, A.: A decision support model for constriction cash flow management. Comput.-Aided Civ. Infrastruct. Eng. 22(7), 527-539 (2007)

9. Mondal, S.R.: Inequalities of extended beta and extended hypergeometric functions. J. Inequal. Appl. 2017, 10 (2017)

10. Olver, F.W.J., Olde Daalhuis, A.B., Lozier, D.W., Schneider, B.I., Boisvert, R.F., Clark, C.W., Miller, B.R., Saunders, B.V. (eds.): NIST Digital Library of Mathematical Functions. http://dlmf.nist.gov/, Release 1.0.22 of 2019-03-15

11. Ravi, B., Venkat, L.A.: Some inequalities for the beta function. Bull. Int. Math. Virtual Inst. 7(3), 403-406 (2017) 